\title{
FOLLOW-UP CONTROL OF A SECOND ORDER SYSTEM IN SLIDING MODE
}

\author{
MÁRK DOMONKOS ${ }^{* 1}$ AND NÁNDOR FINK ${ }^{1}$ \\ ${ }^{1}$ Department of Mechatronics, Optics and Mechanical Engineering Informatics, Budapest University of
Technology and Economics, Bertalan Lajos u. 4-6, Budapest, 1111, HUNGARY
}

\begin{abstract}
This paper deals with an instantaneous feedback controlled inverter using sliding mode control. The theoretical contribution of this paper is that a common state-space-based sliding surface design method is extended for follow-up control. Starting from the basic theory of sliding mode control, the trajectory of the error signal is modelled. The practical contribution of this paper is the relationship between the trajectory of the error signal and the Total Harmonic Distortion (THD) of the output voltage signal. The conditions of the sliding mode control using an Uninterruptible Power Supply (UPS) are discussed. A rectifier as a non-linear load is simulated. Experimentally validated simulation results are presented.
\end{abstract}

Keywords: sliding mode control, hysteresis control, second order system, UPS

\section{Introduction}

Power electronics equipment that produces alternating voltage and current impulses is typical of Variable Structure Systems (VSSs). A VSS usually consists of a state when it is insensitive to variations in parameters and load disturbances. This state is referred to as the sliding mode. The cost of insensitivity is an infinitely high switching frequency. Due to the limits with regard to switching delays and frequencies of controlled switches, an ideal sliding mode does not exist. However, the ideal sliding mode can be approximated with an acceptable degree of accuracy.

The theory of Variable Structure Systems and sliding mode control was developed decades ago in the Soviet Union, mainly by Vadim I. Utkin [1] and David K. Young [2]. According to the theory, sliding mode control should be robust but experiments have shown that it is subject to serious limitations. The main problem with applying the sliding mode is the high frequency of oscillation around the sliding surface, referred to as chattering, which strongly reduces the performance of the control. Very few have managed to put the robust behavior predicted by the theory into practice. Many have concluded that the presence of chattering makes the sliding mode control a good game theory, which is not applicable in practice. Over the following period, researchers invested most of their energy in chattering-free applications, leading to the development of numerous solutions like the discrete-time sliding mode [3], sector sliding mode [4], adaptive sliding mode [5] and terminal sliding mode [6].

\footnotetext{
*Author for correspondence: domonkos@mogi.bme.hu
}

Sliding modes can also be used for feedback compensation [7].

The design of a sliding mode controller consists of three main steps. The first is the design of the sliding surface, the second step is the design of the control law which holds the system trajectory on the sliding surface, and the third and key step is implementation of the chattering-free sliding mode control. The systematic sliding manifold design for linear systems was proposed by Utkin [1]. This method was extended in several ways and optimal sliding manifold designS proposed, e.g. frequency-shaped sliding mode control (FSSMC) [8] surface design based on $H^{\infty}$ control theory $[9,10]$ and Tensor Product Model Transformation-based Sliding Surface Design [11]. The reference signal was constant in all the aforementioned papers. Recently, the application of predictive control has become popular [12]. The new element in this paper is that the original method was extended for Follow-up Control.

According to Refs. [13] and [14], Uninterruptible Power Supplies (UPSs) are being broadly adopted for the protection of sensitive loads, e.g. PCs, air traffic control systems, life care medical equipment, etc., against line failures or other perturbations in AC mains. Ideally, an UPS should be able to deliver: 1) a sinusoidal output voltage with low total harmonic distortion during normal operation, even when feeding nonlinear loads (particularly rectified loads); 2) the voltage dip and recovery time due to a change in load step must be kept as small as possible, that is, provide a fast dynamic response; 3) the steadystate error between the sinusoidal reference and load regulation must be zero. To achieve these, a Proportional In- 
tegral (PI) controller is usually used [15], moreover, issues concerning robust stability and controller robustness are discussed in [16]. However, the system using the PI controller subject to a variable load rather than the nominal ones cannot produce a fast and stable output voltage response. In the literature, some hybrid solutions to overcome this problem can be found $[17,18]$. The principle of Pulse Width Modulation (PWM) plays a very important role in power electronics [19]. In the field of inverter technology, which produces a sinusoidal voltage, a great number of "optimized PWM" techniques have been proposed in the literature. These types of PWM inverters have very good steady-state characteristics, but for the voltage regulator to respond to a sudden change in the load takes a few cycles and nonlinear loads can cause high "load harmonics". This is unacceptable in UPS applications for which instantaneous feedback is preferred [20, 21]. The sliding mode control of power electronic inverters is suggested in Refs. [22] and [23]. On the one hand the main advantage of the sliding mode-based method proposed in this paper is the direct control of the transistor switches, but on the other hand uncontrolled THD results. That is why the THD is the focus of this paper.

The structure of this paper is as follows. After the Introduction, Section 2 presents the problem statement, describes the system configuration and summarizes the design of the sliding mode control. Section 3 describes the equations of the system and shows how the mathematical foundations are applied to a practical example. Following the main steps of sliding mode design, namely the surface design, a control law is selected by taking into consideration the reduced chattering. Section 4 presents the simulation results, which are validated by experimental measurements of a $10 \mathrm{kVA}$ UPS. Finally, in Section 5, the presented results are analyzed.

\section{Problem Statement}

\subsection{System Configuration}

A simplified diagram of the inverter and filter is shown in Fig. $1 V_{\mathrm{b}}$ denotes the battery voltage and $v_{\mathrm{i}}$ stands for the input voltage of the system, which is a filter with a load. The input signal consists of three different values $\left(V_{\mathrm{b}}\right.$, $-V_{\mathrm{b}}$ and 0 ) depending on the switching states of the transistors. The goal is that the transistors must be switched in such a way that $v_{\mathrm{o}}$, the output voltage of the system, follows the sinusoidal reference signal. $L_{\mathrm{s}}$ in Fig. 1 denotes the leakage inductance of the transformer, which has a special structure to increase and set the value of $L_{\mathrm{s}}$. The main field inductance $L_{\mathrm{p}}$ cannot be ignored from the point of view of the resonance circuit. The loss of the transformer is modelled by an increased load.

$$
v_{\mathrm{o}}(t)=\sqrt{2} \cdot 230 \cos (2 \pi 50 t)
$$

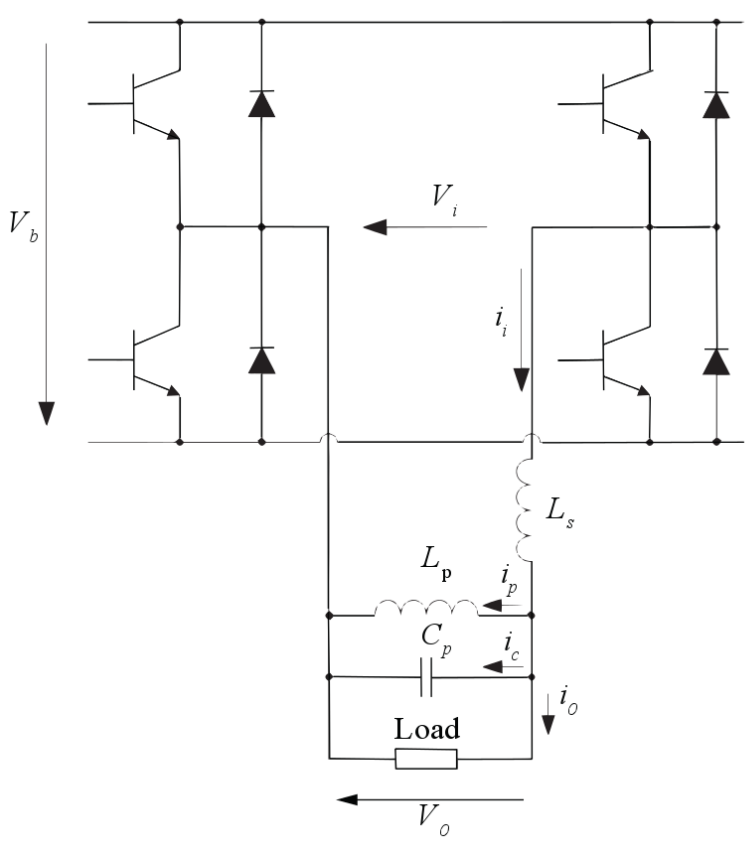

Figure 1: Simplified figure of the inverter and filter

\subsection{Sliding Mode Control}

A Single-Input Single-Output (SISO) system is given in state-space controllable canonical form.

$$
\begin{gathered}
\dot{\mathbf{x}}(t)=\mathbf{A} \mathbf{x}(t)+\mathbf{b} u(t) \\
y(t)=\mathbf{c x}(t)
\end{gathered}
$$

where $\mathbf{x}(t) \in \mathbb{R}^{n}, \mathbf{A} \in \mathbb{R}^{n \times n}, \mathbf{b} \in \mathbb{R}^{n \times 1}, \mathbf{c} \in \mathbb{R}^{1 \times n}$ and $u(t), y(t) \in \mathbb{R}$. $y(t)$, the output variable of the controlled plant, can be described by a $n$-th order differential equation supposing that the reference signal $y_{\mathrm{r}}(t)$ can be differentiated at least $n$ times.

The goal is given in

$$
y_{\mathrm{r}}(t)=y(t) \text { if } t>T_{\mathrm{c}}
$$

where $T_{\mathrm{c}}$ denotes the control period. The system error, $e_{y}(t)$, is given by

$$
e_{y}(t)=y_{\mathrm{r}}(t)-y(t)
$$

Thus the error signal, $e_{y}(t)$, can also be differentiated at least $n$ times, so the $n-1$-th derivative must exist and is continuous. Due to the latter property, when trying to eliminate the error, it is also useful to control its derivatives (including the $n-1$-th derivative). Otherwise, because of the system inertia, an oscillation with a large amplitude could arise. The essence of sliding mode control could be summarized as follows: in order to eliminate the error in the $n$-th dimensional phase space, a continuous error trajectory running into the origin has been designed. Whilst planning, the physical limits should be taken into account. By implementing our very own transient, the ideal system follows the reference signal without any errors. The control signal is designed so that the 
trajectory realized does not deviate from the prescribed one or once the origin has been reached, it remains at rest.

Usually $\sigma$, a scalar variable, can be defined as a positive or negative distance between the desired and actual trajectories, or each trajectory sector can be prescribed by a single scalar variable. The task of the controller is to ensure this scalar variable remains zero. In the classical method of sliding mode control, this scalar variable is calculated as a linear combination of the error and its derivatives [1]. When $n=2$, the scalar variable can be defined by the following equation:

$$
\sigma(t)=e_{y}(t)+\tau \dot{e}_{y}(t)
$$

where $\tau$ denotes a time constant-type control parameter chosen by us. In sliding mode control:

$$
\sigma=0
$$

and the trajectory is described by the following equation:

$$
e_{y}(t)=-\tau \dot{e}_{y}(t)
$$

that corresponds to a $-1 / \tau$ gradient in the phase plane. This is usually referred to as the sliding line or sliding surface in multidimensional phase space. The solution of Eq. 8 is an exponential function with a negative exponent:

$$
e_{y}(t)=E_{0} e^{-\frac{t}{\tau}} \mathrm{t} \geq 0
$$

where $E_{0}=e_{y}(0)$. Consequently, the error will decrease according to the chosen time constant, $\tau$, independently from the system parameters, e.g. load. After an exponential transient process, $y_{\mathrm{r}}(t)=y(t)$. To ensure that the system moves in all cases towards the sliding mode (i.e. towards $\sigma(t)=0$ ), the following condition is necessary:

$$
\sigma(t) \dot{\sigma}(t) \leq 0
$$

The design of a sliding mode controller does not require accurate modelling, it is sufficient to only know the boundaries of the model parameters and the disturbance. During sliding mode control, the only task is to switch the control signal so that Eq. 10 is valid in every instance. No other information concerning the controlled plant and the disturbances is needed. It is sufficient to determine whether Eq. 10 holds or not. In simple cases (or within a range of errors and its derivatives), the signs of the control signal and $\dot{\sigma}(t)$ are opposite. Thus it is often satisfactory to use a relay controller, which switches the control signal according to the sign of the parameter $\sigma(t)$.

\subsection{Follow-up Control}

Since $y_{\mathrm{r}}(t)$ is not constant, $W_{y_{\mathrm{r}}, u}(s)$, the transfer function from $u(t)\left(v_{\mathrm{i}}(t)\right)$ to $y_{\mathrm{r}}(t)\left(v_{\mathrm{o}}(t)\right)$, must be calculated. According to Eq. 4, it is the inverse of the transfer function with regard to the inverted system:

$$
y(s)=W_{u, y}(s) u(s)
$$

$$
W_{y_{\mathrm{r}}, u}(s)=W_{u, y}^{-1}(s)
$$

Usually, the inverse of $W_{u}, y(s)$ cannot be realized. In our case, $y_{\mathrm{r}}(t)$ is sinusoidal:

$$
y_{\mathrm{r}}(t)=\sqrt{2} V_{\mathrm{r}} \cos \left(\omega_{\mathrm{r}} t\right)
$$

Only two parameters must be calculated, namely the gain $\left(G_{\mathrm{r}}\right)$ and phase shift $\left(\varphi_{\mathrm{r}}\right)$ of the transfer function of the system at the reference angular frequency $\left(\omega_{\mathrm{r}}\right)$. The definition of the error (Eq. 5) must be modified:

$$
e_{y}(t)=\frac{\sqrt{2}}{G_{\mathrm{r}}} V_{\mathrm{r}} \cos \left(\omega_{\mathrm{r}} t-\varphi_{\mathrm{r}}\right)-y(t)
$$

\section{Equations of the System}

\subsection{Filter and Load}

Using the notation of Fig. 1, the equation for the currents is:

$$
i_{\mathrm{i}}(t)=i_{\mathrm{o}}(t)+i_{\mathrm{p}}(t)+i_{\mathrm{c}}(t)
$$

By assuming resistive load $R_{\mathrm{L}}$ :

$$
i_{\mathrm{o}}(t)=\frac{1}{R_{\mathrm{L}}} v_{\mathrm{o}}(t)
$$

The equation of the input voltage is

$$
v_{\mathrm{i}}(t)=L_{\mathrm{s}} \frac{\mathrm{d} i_{\mathrm{i}}(t)}{\mathrm{d} t}+v_{\mathrm{o}}(t)
$$

By substituting Eqs. 15 and 16 into Eq. 17, the filter circuit with a resistive load can be described by the following differential equation:

$$
v_{\mathrm{i}}(t)=L_{\mathrm{s}} C_{\mathrm{p}} \ddot{v}_{\mathrm{o}}(t)+\frac{L_{\mathrm{s}}}{R_{\mathrm{L}}} \dot{v}_{\mathrm{o}}(t)+\frac{1}{G} v_{\mathrm{o}}(t)
$$

where

$$
G=\frac{L_{\mathrm{p}}}{L_{\mathrm{s}}+L_{\mathrm{p}}}
$$

The transfer function from $u(t)\left(v_{\mathrm{i}}(t)\right)$ to $y_{\mathrm{r}}(t)\left(v_{\mathrm{o}}(t)\right)$ (including the effect of the resistive load) can be calculated from the Laplace transformed form of Eq. 18:

$$
v_{\mathrm{o}}(s)=\frac{G}{G L_{\mathrm{s}} C_{\mathrm{p}} s^{2}+\frac{G L_{\mathrm{s}}}{R L} s+1} v_{\mathrm{i}}(s)=W(s) v_{\mathrm{i}}(s)
$$

\subsection{The states of the state-space equation}

Since the inverter and load are handled separately, the system has two inputs, namely $u_{\mathrm{i}}$ and $i_{\mathrm{o}}$. Since the input current must be calculated, three state variables are selected for the three storage elements, even if they are not independent and the rank of the system matrix is only 2. Using the notation of Fig. 1, the three state variables are $i_{\mathrm{i}}(t), i_{\mathrm{p}}(t)$ and $v_{\mathrm{c}}(t)$ :

$$
\mathbf{x}(t)=\left[\begin{array}{c}
i_{\mathrm{i}}(t) \\
i_{\mathrm{p}}(t) \\
v_{\mathrm{o}}(t)
\end{array}\right]
$$


The load is connected in parallel to the inverter capacitor $C_{\mathrm{p}}$. The load current includes the effect of transformer losses. The system has three outputs since $v_{\mathrm{o}}(t)=v_{\mathrm{c}}(t)$ as well as its derivative are necessary to calculate the scalar variable and $i_{\mathrm{i}}(t)$ is visualised. In the real system, the current of the capacitor is measured instead of $\dot{v}_{\mathrm{o}}(t)$. According to Eqs. 15, 16, and 17, the matrices of the system are:

$$
\begin{aligned}
\boldsymbol{A}=\left[\begin{array}{ccc}
0 & 0 & -\frac{1}{L_{\mathrm{s}}} \\
0 & 0 & \frac{1}{L_{\mathrm{p}}} \\
\frac{1}{C_{\mathrm{p}}} & -\frac{1}{C_{\mathrm{p}}} & 0
\end{array}\right] & \boldsymbol{B}=\left[\begin{array}{cc}
0 & -\frac{1}{L_{\mathrm{s}}} \\
0 & 0 \\
-\frac{1}{C_{\mathrm{p}}} & 0
\end{array}\right] \\
\left.\begin{array}{ccc}
1 & 0 & 0 \\
0 & 1 & 0 \\
0 & 0 & 1
\end{array}\right] & \boldsymbol{D}=\left[\begin{array}{ll}
0 & 0 \\
0 & 0 \\
0 & 0
\end{array}\right]
\end{aligned}
$$

\subsection{Error Trajectory}

To calculate the error defined by Eq. $14, G_{\mathrm{r}}$ and $\varphi_{\mathrm{r}}$ must first be calculated. According to Eq. 20:

$$
W\left(j \omega_{\mathrm{r}}\right)=\frac{G}{G L_{\mathrm{s}} C_{\mathrm{p}}\left(j \omega_{\mathrm{r}}\right)^{2}+\frac{G L_{\mathrm{s}}}{R L} j \omega_{\mathrm{r}}+1}
$$

$G_{\mathrm{r}}$ and $\varphi_{\mathrm{r}}$ can be calculated from Eq. 23 , where $W\left(j \omega_{\mathrm{r}}\right)$ denotes a simple complex number:

$$
\begin{gathered}
G_{\mathrm{r}}=\left|W\left(j \omega_{\mathrm{r}}\right)\right| \\
\varphi_{\mathrm{r}}=\operatorname{angle}\left(W\left(j \omega_{\mathrm{r}}\right)\right)
\end{gathered}
$$

Since the filter must be inductive, $\varphi_{\mathrm{r}}$ must be negative. Therefore, the reference signal must lead to $v_{\mathrm{o}}(t)$, which is the controlled signal in Eq. 14:

$$
y(t)=v_{\mathrm{o}}(t)
$$

The derivative of the error is:

$$
\dot{e}_{y}(t)=-\omega_{\mathrm{r}} \frac{\sqrt{2}}{G_{\mathrm{r}}} V_{\mathrm{r}} \sin \left(\omega_{\mathrm{r}} t-\varphi_{\mathrm{r}}\right)-\dot{v}_{\mathrm{o}}(t)
$$

The scalar variable of the sliding mode control is calculated by Eq. 6 .

\subsection{Control Law}

Three different control laws were examined. All of them can directly control the switching of the transistors. This is the main advantage of sliding mode control in the field of power electronics. More advanced controllers can be found in Ref. [24].

\section{Simple Relay}

The input voltage on the filter is switched according to the sign of $\sigma(t)$ :

$$
v_{\mathrm{i}}(t)=V_{\mathrm{b}} \operatorname{sign}(\sigma(t))
$$

From a practical point of view, the main problems of Eq. 28 are

- the inverter has a zero state, which is not applied;

- the switching frequency is uncontrolled.

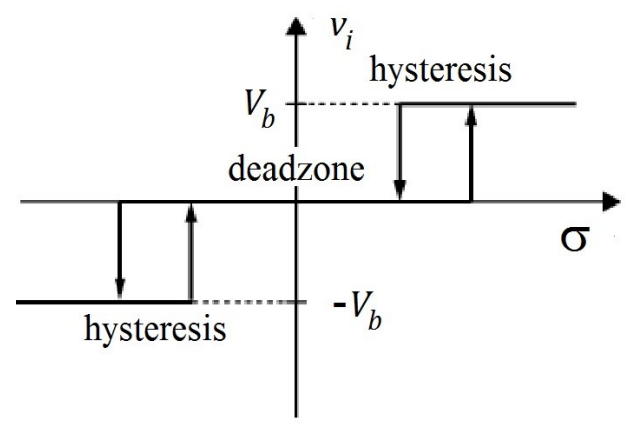

Figure 2: Double Relay with dead zone and hysteresis

\section{Double Relay}

The solution to the first problem is the usage of two relays:

$$
v_{\mathrm{i}}(t)=\frac{V_{\mathrm{b}}}{2} \operatorname{sign}(\sigma(t))+\frac{V_{\mathrm{b}}}{2} \operatorname{sign}\left(\cos \left(\omega_{\mathrm{r}} t-\varphi_{\mathrm{r}}\right)\right)
$$

$V_{\mathrm{b}}$ and 0 are switched in the positive half period, while $-V_{\mathrm{b}}$ and 0 are switched in the negative half period. However, Eq. 29 does not solve the second problem.

\section{Double Relay with dead zone and hysteresis}

Both problems can be solved by a double relay with dead zone and hysteresis. This control law is shown in Fig. 2.

\subsection{Stability Analysis}

Using a simple relay controller, whether or not Eq. 10 holds can be verified. First, $\dot{\sigma}(t)$ must be expressed:

$$
\dot{\sigma}(t)=\dot{e}_{y}(t)+\tau \ddot{e}_{y}(t)
$$

According to Eq. 27:

$$
\ddot{e}_{y}(t)=-\omega_{\mathrm{r}}^{2} \frac{\sqrt{2}}{G_{\mathrm{r}}} V_{\mathrm{r}} \cos \left(\omega_{\mathrm{r}} t-\varphi_{\mathrm{r}}\right)-\ddot{v}_{\mathrm{o}}(t)
$$

$\ddot{v}_{\mathrm{O}}(t)$ can be expressed from Eq. 18:

$$
\ddot{v}_{\mathrm{o}}(t)=\frac{1}{L_{\mathrm{s}} C_{\mathrm{p}}} v_{\mathrm{i}}(t)-\frac{1}{R_{\mathrm{L}} C_{\mathrm{p}}} \dot{v}_{\mathrm{o}}(t)-\frac{1}{G L_{\mathrm{s}} C_{\mathrm{p}}} v_{\mathrm{o}}(t)
$$

By substituting Eq. 28 into Eq. 32:

$$
\ddot{v}_{\mathrm{o}}(t)=\frac{V_{\mathrm{b}} \operatorname{sign}(\sigma(t))}{L_{\mathrm{s}} C_{\mathrm{p}}}-\frac{\dot{v}_{\mathrm{o}}(t)}{R_{\mathrm{L}} C_{\mathrm{p}}}-\frac{v_{\mathrm{o}}(t)}{G L_{\mathrm{s}} C_{\mathrm{p}}}
$$

According to Eqs. 30 and 31, if the value of $\ddot{v}_{\mathrm{o}}(t)$ is sufficiently large and the system operates in an area which is close enough to the sliding mode, then the sign of $\ddot{v}_{\mathrm{o}}(t)$ is the opposite to that of $\dot{\sigma}(t)$. From Eq. 33, it is clear that if the value of $V_{\mathrm{b}}$ is sufficiently large and the system operates in an area which is close enough to the sliding mode, then the sign of $\ddot{v}_{\mathrm{o}}(t)$ is the same as that of $\sigma(t)$. In conclusion, if the value of $V_{\mathrm{b}}$ is sufficiently large and the 
system operates in an area which is close enough to the sliding mode, then the sign of $\sigma(t)$ is the opposite to that of $\dot{\sigma}(t)$. Similar analyses can be conducted for all control laws. In practice, the simulations proved that by substituting the actual parameters of the system, all the control laws examined fulfil the condition (Eq. 10) during normal operations.

\section{Simulation}

MATLAB-Simulink simulations were carried out.

\subsection{MATLAB-Simulink model}

The main elements of the MATLAB-Simulink model are shown in Figs. 3-6. Even if the structure of the controller shown in Fig. 6 is quite simple, its performance is quite robust.

\subsection{Model validation}

The simulation results were compared to previous results measured by [25] as shown in Fig. 7. The nominal power of the inverter is 10 VA. All parameters of (14) and (22) in addition to the values of dead zone and hysteresis shown in Fig. 2 are known from Ref. [25].

Like in real systems, where $\sigma$ is tuned manually to set the number of switches per period, $\sigma$ is changed to achieve a similar result in the simulation as in real systems. The simulation result is shown in Fig. 8 after the value of $\sigma$ was set.

The first problem faced in the simulation was the steady state. When the real system was measured, the screen of the oscilloscope seemed to be frozen for most settings of $\sigma$. Therefore, the error trajectory was a closed curve during a period in a steady state.

Using the default settings of Simulink, a quasi-steadystate period of the error trajectory of the simulation resulted as is shown in Fig. 9. The consequent periods are slightly different, which causes subharmonics that are crucial from a practical point of view.

The main question is whether these subharmonics are an immanent property of the applied switching method or a result of an insufficiently precise simulation. Several simulations were carried out using different model configuration parameters in Simulink. It is concluded that the precise calculation of the switching instant is key to simulating proper steady states. The Ode113(Adam) method is the most suitable integral method for switched systems. (The equation solvers for UPS are compared in Ref. [26]).

\subsection{Analysis of the simulation results}

The main advantage of the PWM technique is that the filter can be smaller resulting in a smaller no-load current. The parameters of the filter are given in

$$
L_{\mathrm{s}}=3.5 \mathrm{mH}, L_{\mathrm{p}}=32 \mathrm{mH}, C_{\mathrm{p}}=320 \mu \mathrm{F}
$$

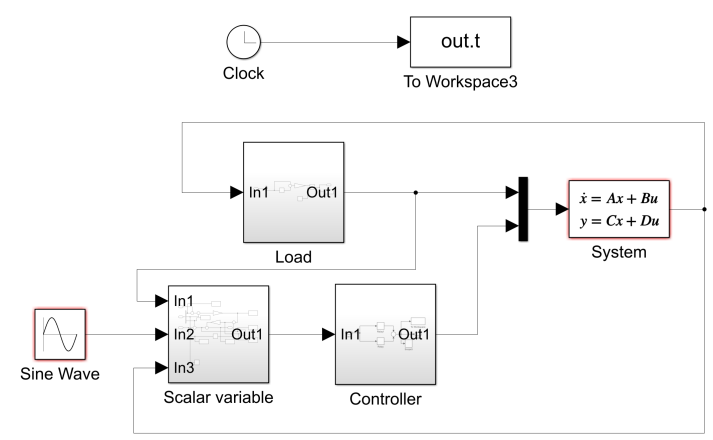

Figure 3: The whole system.

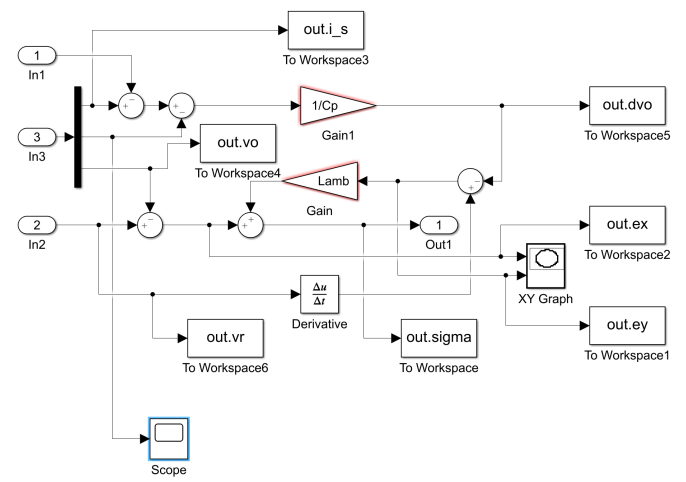

Figure 4: Subsystem for calculating scalar variableS.

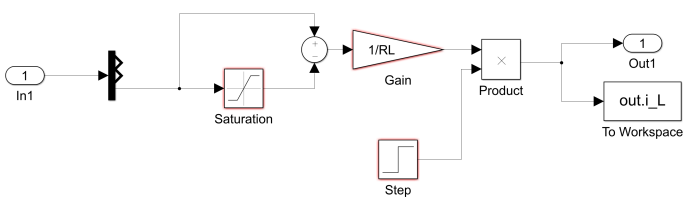

Figure 5: Subsystem for the load.

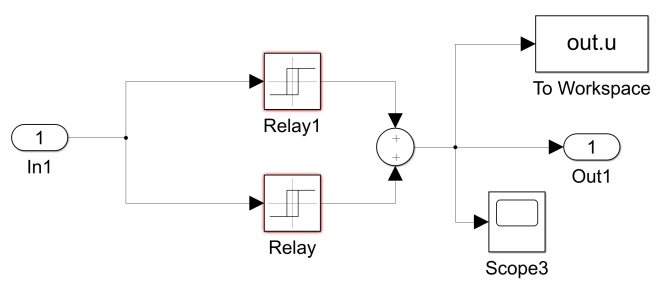

Figure 6: Subsystem for the relay controller of the dead zone and hysteresis

$\tau$, the gradient of the switching line in the sliding mode, has been changed. The value of the nominal load is $R_{\mathrm{L}}=5.3 \Omega$. The relationship between the gradient of the switching line and the number of switches is shown in Fig. 10. The larger the value of $\tau$, the smaller the gradient of the switching line and the greater the number of switches per period. On the other hand, the bigger the value of $\tau$, the longer the transient according to Eq. 9. The optimum must be calculated.

The number of switches per period and the total harmonic distortion (THD) are shown in Fig. 11 as a function of $\tau$. The first ONE is staggered. The larger the num- 


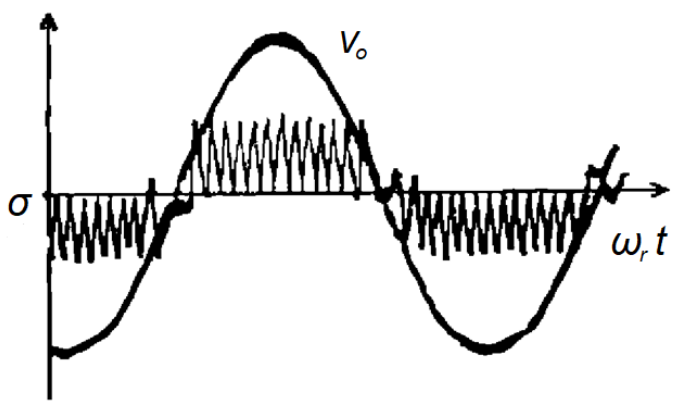

Figure 7: Reference measurement result

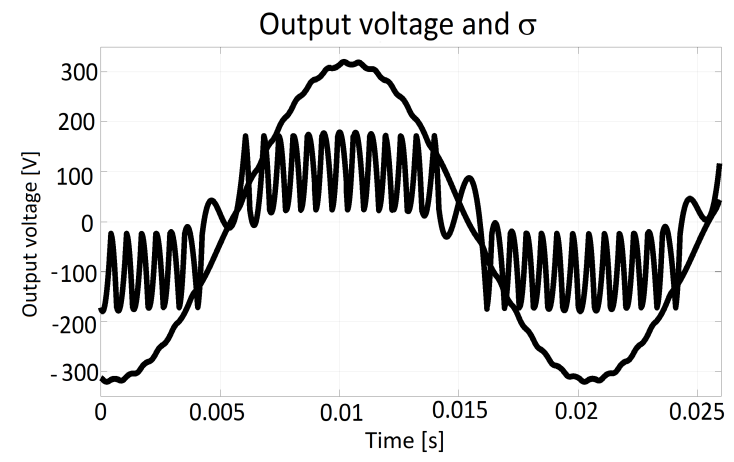

Figure 8: Reference simulation result

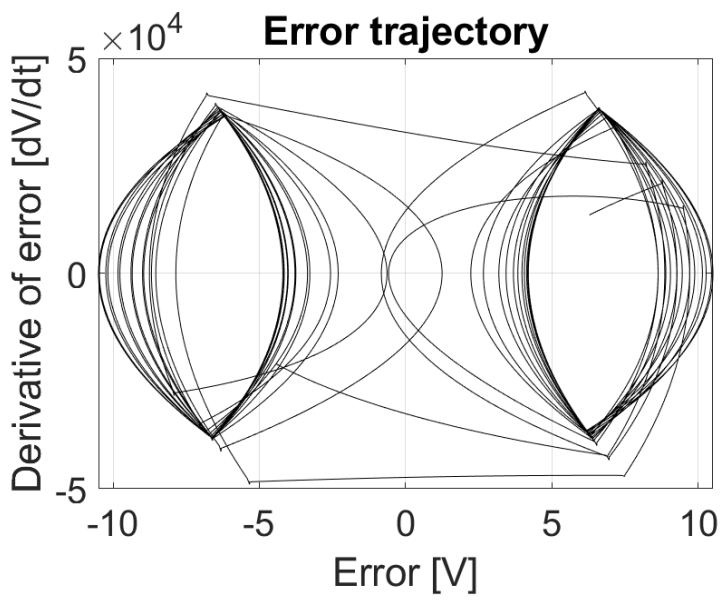

Figure 9: Error trajectory during a quasi-steady-state period

ber of switches is, the greater the switching loss and the smaller the THD are. The optimum is approximately 52 switches, therefore, 13 pulses per period, as is shown in Fig.7 and Fig. 8. The step concerning the optimal number of switches is magnified in Fig. 12.

A local minimum of THD is located at the middle of a staircase (in Fig. 12) when the transient between the positive and negative half periods is smooth as is shown in Fig. 13.

By increasing $\tau$, the transients change slightly. First, a V shape appears (see Fig. 14).

By further increasing $\tau$, the $\mathrm{V}$ shape transforms into a loop (see Fig. 15) and the last pulses in all half periods have the opposite signs when compared to the reference signal (see Fig. 16) which increases the THD. Finally, the number of switches per period is increased by 4 and the

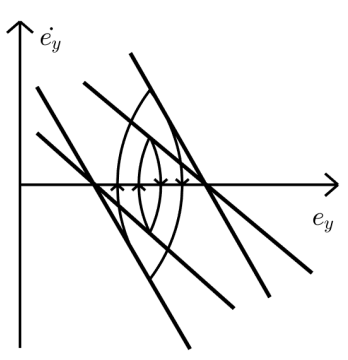

Figure 10: The effect of $\tau$ on the number of switches

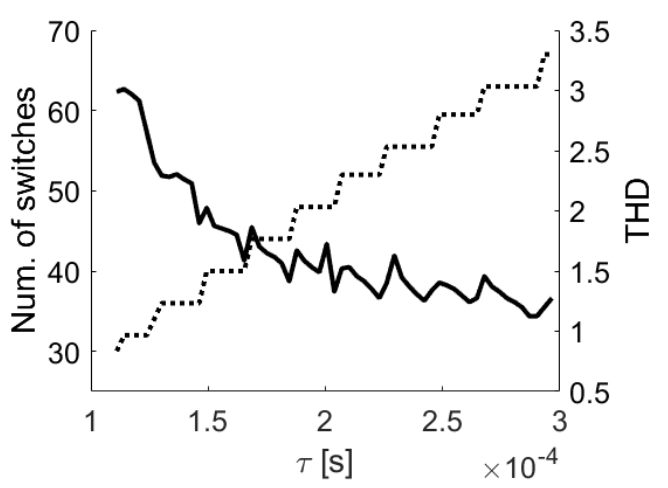

Figure 11: Number of switches and THD as a function of the gradient of the switching line

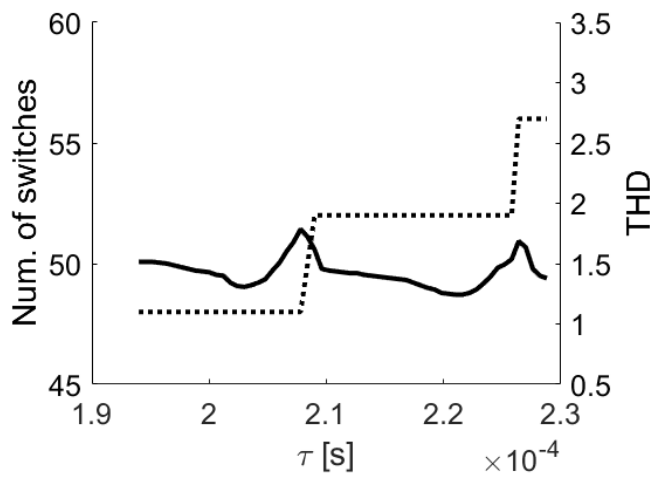

Figure 12: The optimal number of switches and its THD

transients are in the form of Vs and loops once more (see Fig. 17), which disappear at the middle of the staircase.

\subsection{Non-linear load}

The most challenging load is the rectifier [27], since its current is not sinusoidal and the value of its peak is relatively large. A rectifier resembling a load is simulated, which has approximately the same root mean square (RMS) value of the current as that of the nominal current. The time functions of the output voltage and current in addition to the input current are shown in Fig. 18. The RMS value of the input no-load current is zero at the beginning of the period, when the output current is zero. Since the load current is continuous and the reaction of the sliding mode controller is practically instantaneous as a result, this type of non-linear load has no significant ef- 


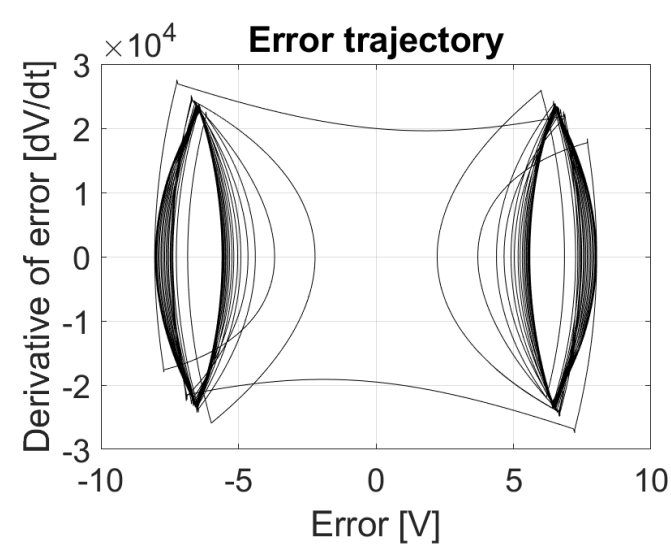

Figure 13: Smooth transient of error trajectory

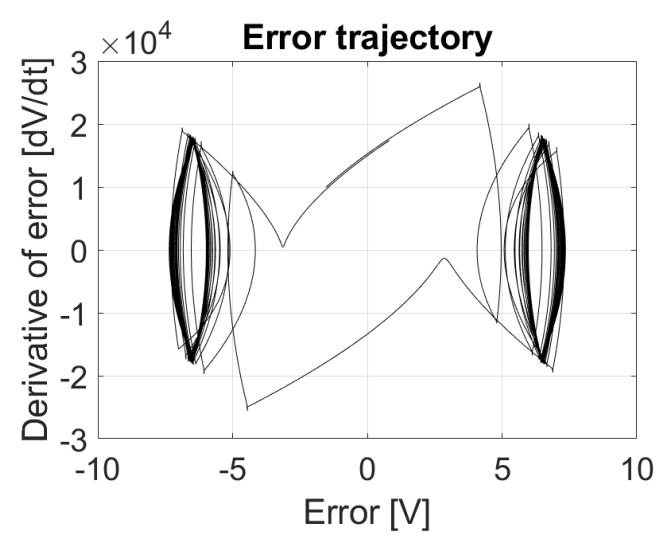

Figure 14: V-shaped transient of error trajectory

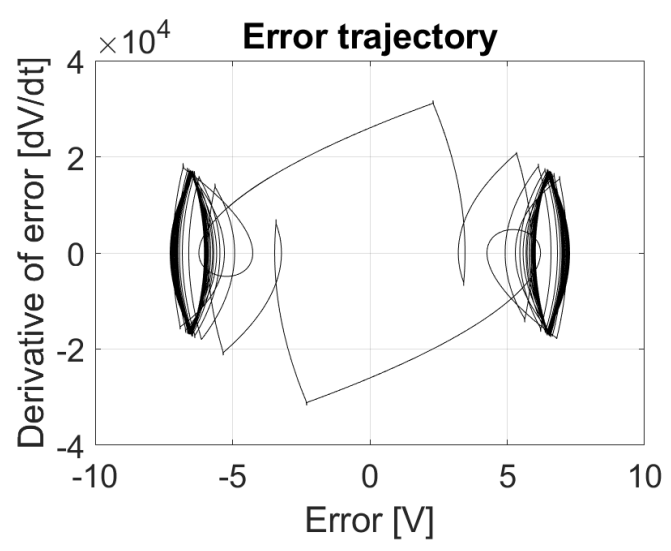

Figure 15: Loop-shaped transient of error trajectory

fect on the output voltage - the THD depends on the shape of the error trajectory, which is shown in Fig. 19:

\subsection{Switching on the load}

A nominal resistive load is assumed and the load is switched on at the peak value of the output voltage. The time functions of the voltage and current are shown in Fig. 20. Since the current is discontinuous, a transient is

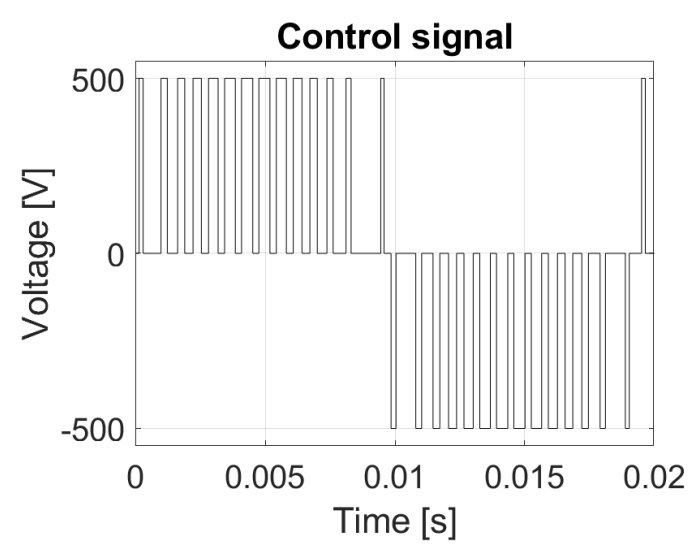

Figure 16: Control signal

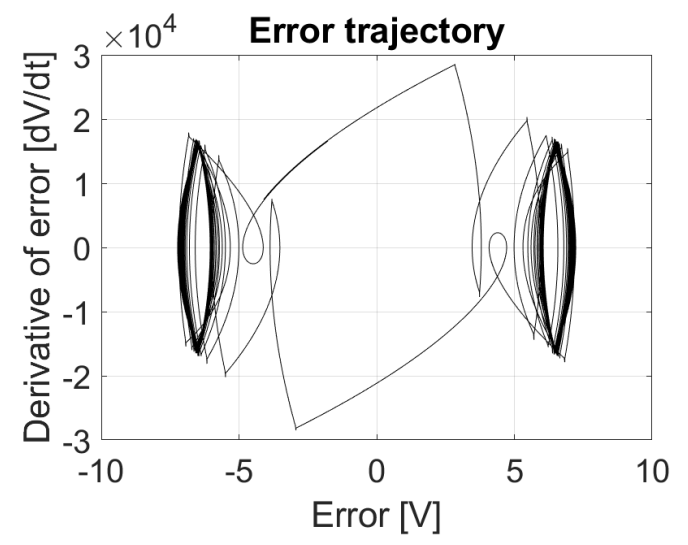

Figure 17: Transient of error trajectory after the number of switches is increased

present, which is recognizable in terms of the shape of the error trajectory in Fig. 21. The length of the transient depends on the value of $\tau$, that is, $0.22 \mathrm{~ms}$. The transient caused by any step change in the load occurs extremely quickly and only lasts approximately $1 \mathrm{~ms}$.

\section{Conclusions}

This paper introduced a follow-up control method with a sinusoidal reference signal for the design of a sliding surface and the performance of a Double Relay controller with a dead zone and hysteresis operating in a sliding mode was analysed. The simulation of variable structure systems is very sensitive to the integral method of simulation. The zero-crossing must be detected. Even a relatively simple controller, which can be implemented by an analogue operational amplifier, also performs very well with a non-linear load. The positive and negative half periods can be separated by a dead zone and the switching frequency limited by hysteresis. The actual number of switches can be set by the gradient of the sliding line. The value of the THD depends on the shape of the error phase trajectory. A non-linear load with a continuous output current has no significant effect on the output voltage because of the instantaneous behavior of the sliding mode controller. 


\section{Output voltage, input and output current}

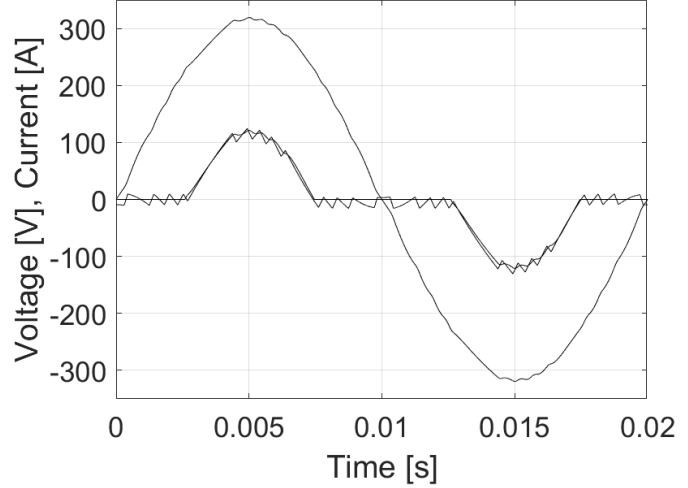

Figure 18: Output voltage as well as input and output currents with a rectifier-like load

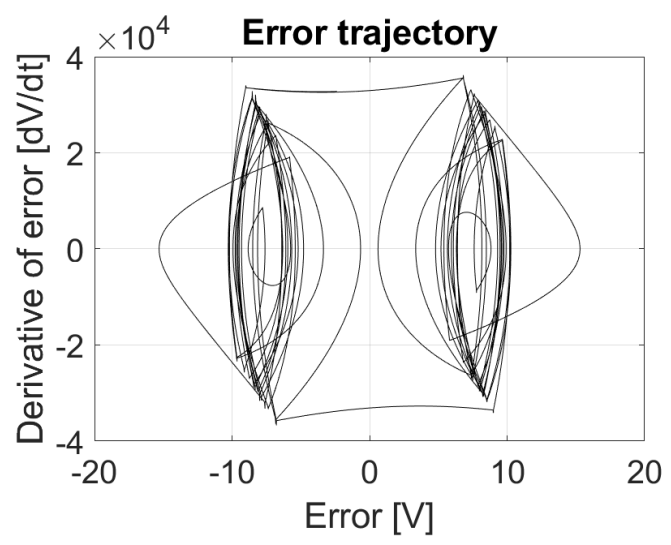

Figure 19: Error trajectory of the non-linear rectifier-like load

\section{Acknowledgement}

The research reported in this paper and carried out at the Budapest University of Technology and Economics has been supported by the National Research Development and Innovation Fund (TKP2020 Institution Excellence Subprogram, Grant No. BME-IE-MIFM) based on the charter of bolster issued by the National Research Development and Innovation Office under the auspices of the Ministry for Innovation and Technology.

\section{REFERENCES}

[1] Utkin, V. I.: Variable structure control optimization (Springer-Verlag), 1992 DOI: 10.1007/978-3-642-84379-2

[2] Young, K.: Controller design for manipulator using theory of variable structure systems, IEEE Trans. Syst. Man Cybern. Syst., 1978, 8(2), 101-109 DOI: 10.1109/TSMC.1978.4309907

[3] Korondi, P.; Hashimoto, H.; Utkin, V.: Discrete sliding mode control of two mass system, in: Proceedings of the 1995 IEEE International Symposium on Industrial Electronics, ISIE' 95 (Piscataway (NJ), USA), 338-343 DOI: 10.1109/ISIE.1995.497019

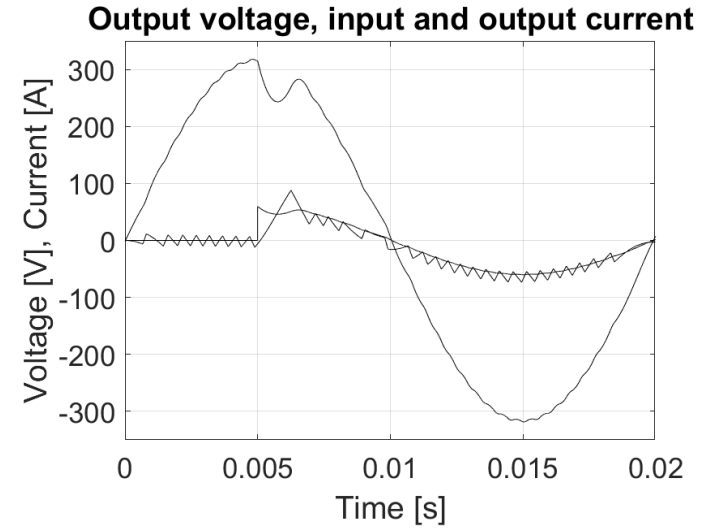

Figure 20: Output voltage as well as input and output currents after switching on the resistive load

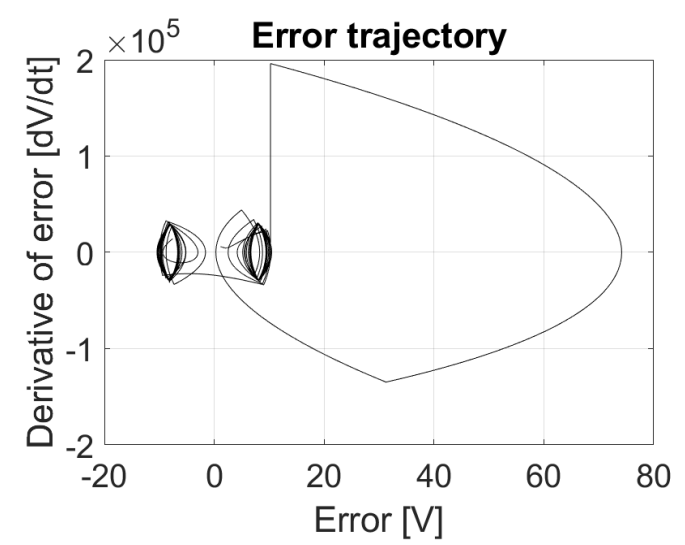

Figure 21: Error trajectory after switching on the resistive load

[4] Korondi, P.; Xu, J., Hashimoto, H.: Sector sliding mode controller for motion control, in: Proceedings of the 8th international power electronics \& motion control conference (PEMC, Prague, Czech), 254 259

[5] Yu, H.; Ozguner, U.: Adaptive seeking sliding mode control, in IEEE American Control Conference (Minneapolis, USA), 2006 DOI: 10.1109/ACC.2006.1657462

[6] Chang, E.-C.; Chang, F.-J.; Liang, T.-J.; Chen, J.F.: DSP-based implementation of terminal sliding mode control with grey prediction for UPS inverters, the 5th IEEE Conference on Industrial Electronics and Applications (ICIEA), 2010, 1046-1051 DOI: 10.1109/ICIEA.2010.5515790

[7] Fink, N.: Model reference adaptive control for telemanipulation, Hung. J. Ind. Chem., 2019, 47(1), 4148 DOI: 10.33927/hjic-2019-07

[8] Young, K.; Özgüner, U.: Frequency shaped sliding mode synthesis, in International Workshop on VSS and Their Applications (Sarajevo)

[9] Fodor, D.; Tóth, R.: Speed sensorless linear parameter variant $H^{\infty}$ control of the induction motor, in Proceedings of the 43rd IEEE Conference Decision 
and Control (IEEE, Piscataway, USA), 4435-4440 DOI: $10.1109 / C D C .2004 .1429449$

[10] Hashimoto, H.; Konno, Y.: Sliding surface design in the frequency domain, in: Variable structure and Lyapunov control , 75-86 (Springer-Verlag), 1993 DOI: $10.1007 / \mathrm{BFb} 0033679$

[11] Korondi, P.: Tensor product model transformationbased sliding surface design, Acta Polytech. Hung., 2006, 3(4), 23-36

[12] Neukirchner, L. R.; Magyar, A.; Fodor, A.; Kutasi, N.D.; Kelemen, A.: Constrained predictive control of three-phase buck rectifiers, Acta Polytech. Hung., 2020, 17(1), 41-60 DOI: 10.12700/APH.17.1.2020.1.3

[13] Tai, T.-L.; Chen, J.-S.: UPS Inverter design using discrete-time sliding-mode control scheme, IEEE Trans. Ind. Electron., 2002, 49(1), 67-75 DOI: 10.1109/41.982250

[14] Fedak, V.; Bauer, P.; Hájek, V.; Weiss, H.; Davat, B.; Manias, S.; Nagy, I.; Korondi, P.; Miksiewicz, R.; Duijsen, P.; Smékal, P.: Interactive E-learning in electrical engineering, in: 15 th International Conference on Electrical Drives and Power Electronics (EDPE) (Podbanské), 368-373

[15] Caceres, R.; Rojas, R.; Camacho, O.: Robust PID control of a buck-boost DC-AC converter, Proc. IEEE INTELEC'02, 2000, 180-185 DOI: 10.1109/intlec.2000.884248

[16] Precup, R.-E.; Preitl, S.: PI and PID controllers tuning for integral-type servo systems to ensure robust stability and controller robustness, Electr. Eng., 2006, 88(2), 149-156 DOI: 10.1007/s00202-004-0269-8

[17] Al-Hosani, K.; Malinin, A.; Utkin, V. I.: Sliding mode PID control of buck converters, European Control Conference, 2009 DOI: 10.23919/ecc.2009.7074821

[18] Li, H.; Ye, X.: Sliding-mode PID control of DCDC converter, 5th IEEE Conference on Industrial Electronics and Applications (ICIEA), 2010, 730734 DOI: 10.1109/ICIEA.2010.5516952
[19] Holtz, J.: Pulsewith modulation - A Survey, IEEE Trans. Ind. Electron., 1992, 39(5), 410-420 DOI: 10.1109/41.161472

[20] Kawamura, A.; Hoft, R.: Instantaneous feedback controlled PWM inverter with adaptive hysteresis, IEEE Trans. Ind. Appl., 1984, 20(4), 769-775 DOI: 10.1109/TIA.1984.4504486

[21] Aamir, M.; Kalwar, K. A.; Mekhilef, S.: Review: Uninterruptible power supply (UPS) system, Renew. Sust. Energ. Rev., 2016, 58, 1395-1410 DOI: 10.1016/j.rser.2015.12.335

[22] Korondi, P.; Hashimoto, H.: Park vector based sliding mode control of UPS with unbalanced and nonlinear load, in: Variable Structure Systems, Sliding Mode and Nonlinear Control (Springer, London, UK), 193-209 DOI: 10.1007/bfb0109978

[23] Korondi, P.; Yang, S. H.; Hashimoto, H.; Harashima, F.: Sliding mode controller for parallel resonant dual converters, J. Circ. Syst. Comp., 1995, 5(4), 735-746 DOI: 10.1142/s0218126695000424

[24] Chang, E.-C.: Study and application of intelligent sliding mode control for voltage source inverters, Energies, 2018, 11(10) 2544 DOI: 10.3390/en11102544

[25] Széll, K.; Korondi, P.: Mathematical basis of sliding mode control of an uninterruptible power supply, Acta Polytech. Hung., 2014, 11(3), 87-106 DOI: 10.12700/APH.11.03.2014.03.6

[26] Keiel, G.; Flores, J. V.; Pereira, L. F. A.; Salton, A. T.: Discrete-time multiple resonant controller design for uninterruptible power supplies, IFAC-PapersOnLine, 2017, 50(1), 6717-6722 DOI: 10.1016/j.ifacol.2017.08.1169

[27] Khan, H. S.; Aamir, M.; Ali,M.; Waqar, A.; Ali, S. U.; Imtiaz, J.: Finite control set model predictive contol for parellel connected online UPS system under unbalanced and nonlinear load, Energies, 2019, 12(4), 581 DOI: 10.3390/en12040581 